\title{
B-learning como aliado ante incapacidades laborales temporales
}

\author{
Gabriel Arturo Lugo Morales*, Dora Luz González-Bañales** \\ Departamento de Sistemas y Computación, Instituto Tecnológico de Durango, Tecnológico Nacional \\ de México.*alugo@itdurango.edu.mx,**doraglez@itdurango.edu.mx
}

\begin{abstract}
Resumen
El presente trabajo muestra el caso en el que un profesor de educación superior enfrentó el reto de no interrumpir su labor de enseñanza ante los problemas derivados de un tratamiento de quimio y radioterapia debido a un Linfoma de Hodgkin. El problema de salud le derivó en incapacidades laborales temporales. El profesor cuyo caso se describe en este trabajo, logró encontrar en las Tecnologías de Información y Comunicaciones (TIC) un aliado para no afectar el semestre académico de sus estudiantes, siendo el B-Learning (Blended learning) un elemento facilitador en su labor docente dado que se trata de un modelo semipresencial de enseñanza-aprendizaje. Las estrategias didácticas incluyeron principalmente videos producidos por el propio profesor, un grupo privado en Facebook, asi como trabajo en equipo. Las estrategias B-Learning seguidas le permitieron al profesor cumplir la totalidad del temario de sus cursos académicos e incluso repercutió en un mejor aprovechamiento académico de sus estudiantes.
\end{abstract}

Palabras clave: B-learning, TIC, Incapacidad laboral, Aprendizaje activo.

\section{Introducción}

Las TIC en la educación representan una oportunidad para innovar incursionando en diferentes modalidades educativas como el aprendizaje activo o el e-learning, buscando una mayor participación del alumno en la adquisición de conocimiento, ya que como lo comenta Lozano (2009 Citado en (Concha Vergara, 2014, pág. 116)). "Una sencilla definición de e-learning es la formación que se imparte mediante el uso de las nuevas tecnologías, por tanto, su distinción respecto con la educación tradicional se centra justamente en la enorme potencialidad y oportunidades que nos ofrecen las TIC para ser usadas como medio excelente para formar a las personas".

Por otro lado para el Centro de Desarrollo Docente e Innovación Educativa (2012, Citado en (Lugo Morales \& González Bañales, 2016, pág. 619)): “el aprendizaje activo se define 
como una estrategia de enseñanza-aprendizaje cuyo diseño e implementación se centra en el alumno al promover su participación y reflexión continuas a través de actividades que promueven diálogo, colaboración, desarrollo y construcción de conocimientos, así como habilidades y actitudes. Las actividades son motivadoras y retadoras, orientadas a profundizar en el conocimiento y desarrollar habilidades de búsqueda, análisis y síntesis de la información, y promover una adaptación activa a la solución de problemas. Se caracteriza por actividades bien estructuradas, con la suficiente flexibilidad para adaptarlas a las características del grupo de aprendizaje e incluso a nivel individual".

Una ventaja adicional de las TIC, la que más se trata de resaltar en este trabajo, es que representan una oportunidad para que el aprendizaje no requiera de la presencia física de los involucrados al mismo tiempo, al permitirse que, de manera asíncrona, las actividades se realicen cuando cada uno de ellos tenga oportunidad. Este valor agregado puede ser aprovechado en los casos en los que alguno de los participantes esté impedido para asistir a una clase presencial.

Con base en lo anterior, la experiencia que aquí se presenta describe la situación de un docente que, debido a problemas de salud, tuvo restricciones para asistir de manera regular a impartir su cátedra, por lo que recurrió al uso de recursos tecnológicos para grabar las clases en video y publicarlas en una red social (Facebook) para que sus alumnos pudieran recibir la instrucción incluso sin la presencia del profesor y continuaran con la adquisición del conocimiento a pesar de las circunstancias.

Al combinar clases presenciales (cuando la salud del profesor así lo permitía) y clases virtuales (cuando no), la modalidad educativa empleada se conoce como Blended learning (B-learning), una forma semipresencial que combina las ventajas de ambas situaciones. El caso se dio durante dos periodos, parcialmente en el semestre enero-junio 2017, de mayo a junio, y totalmente en el semestre agosto-diciembre 2017. En ambos lapsos al impartir la materia de Sistemas Operativos correspondiente al tercer semestre de la carrera de Ingeniería en Sistemas Computacionales en el Instituto Tecnológico de Durango (México), con un total de 28 y 36 alumnos respectivamente.

\section{Metodología}

Este trabajo ha sido desarrollado bajo la Metodología de Sistematización de Experiencias Educativas Innovadoras de la UNESCO (Mogollón, 2016). A continuación, se mencionan algunas definiciones que sustentan teóricamente este proyecto.

El proceso enseñanza-aprendizaje tradicional implica una responsabilidad total del profesor en cuanto a la exposición de los temas y una participación receptiva por parte del alumno, como lo mencionan Maldonado y Juárez (2006): "La enseñanza se considera como un 
proceso lineal y acumulativo que va del profesor al alumno. De esta manera, cuando un profesor enseña, se encuentra implícito el hecho de que los alumnos aprenden”.

Este modelo ha ido cambiando al pasar el tiempo ocasionando cambios en los roles, dejando al docente como facilitador y exigiendo una mayor participación del estudiante, lo cual "conlleva necesariamente una transformación de las prácticas docentes como son: el cambio de actitud del docente, la creación de entornos de aprendizaje, el apoyo a los alumnos a desarrollar su aprendizaje y la construcción del conocimiento de manera crítica" (Maldonado \& Juárez, 2006).

Dentro de esas prácticas docentes transformadas se puede mencionar el B-learning que es "un modelo de enseñanza aprendizaje que incluye tanto formación presencial como elearning, beneficiándose de las ventajas de ambos ((López, 2006) Citado en (Durán, Costaguta, \& Gola, 2011)). Sin embargo, no se trata solo de agregar tecnología a las clases, sino de reemplazar algunas actividades de aprendizaje con otras apoyadas con tecnología ((Rosas, 2005) Citado en (Durán, Costaguta, \& Gola, 2011)). Los sistemas basados en Blearning se caracterizan por la flexibilidad e interactividad que facilitan los recursos de información y las herramientas de comunicación (Chat, foro, correo electrónico, etc.), lo cual permite superar, por ejemplo, los obstáculos que aparecen en el desarrollo de trabajos grupales presenciales ((Sánchez, 2005) Citado en (Durán, Costaguta, \& Gola, 2011)), obstáculos tales como las limitaciones de tiempo y espacio para las reuniones, el no poder compartir la información simultáneamente, entre otros (Durán, Costaguta, \& Gola, 2011).

\section{Proceso de planificación e implementación de estrategias B-learning}

\subsection{Antecedentes}

Detallando la situación bajo estudio, al docente involucrado se le diagnosticó cáncer (Linfoma de Hodgkin) en febrero de 2017, iniciando tratamiento de quimioterapia en marzo del mismo año.

Los efectos secundarios del tratamiento complicaban en ocasiones la asistencia al aula pero a pesar de ello, el profesor siguió impartiendo su clase de forma presencial. Otra situación que hacía más difícil la impartición de la clase en el salón fue que, debido a la quimioterapia, el sistema inmunológico del profesor resultó afectado, por lo que se debía restringir el acceso al aula a personas que presentaran síntomas de enfermedades infectocontagiosas, como un resfriado o padecimientos similares que comprometieran el estado de salud del docente, esto generó fricciones con algunos estudiantes, pues pretendían asistir a clase aun estando enfermos, sin importar las posibles consecuencias (el médico 
tratante advirtió al paciente que un resfriado en una persona común puede convertirse en neumonía en una persona inmunodeprimida), llegando al extremo en que un alumno se sentó justo frente al profesor, estornudando y aseándose la nariz a muy corta distancia, esta circunstancia fue la que detonó la búsqueda de una alternativa a través del uso de las TIC y se decidió experimentar con el modelo B-learning a partir de mayo de 2017, es decir, el $60 \%$ de la clase se impartió de manera presencial y el resto de manera semipresencial.

\subsection{Organización de los contenidos de clase}

Se probaron varios programas de captura de pantalla en video, término conocido como screencast para producir "Objetos de Aprendizaje (OAs) y Objetos Didácticos Audiovisuales (ODAs)" mediante grabaciones de audio-video en la pantalla del ordenador (Mirete, Maquilón, \& Sánchez, 2014), entre ellos Camtasia, que resultó muy "pesado" de descargar y además tiene costo.

Después se experimentó con el software Free Screen Video Recorder, funcionando bien por algún tiempo pero luego los productos obtenidos mostraban desfase entre audio y video por lo cual se intentaron otras opciones dentro de las cuales se puede mencionar el sitio web screencast-o-matic.com, que en su versión sin costo tiene límite de 15 minutos por video, limitante que no representaba problema pero sí lo fue el tiempo para generación del archivo correspondiente, alrededor de otros 15 minutos, lo cual consumía aproximadamente 30 minutos por cada video y esto afectaba la productividad pues el tiempo promedio total de una sesión en video comprendía cerca de 40 minutos, lo que implicaba un total de 80 minutos para tener una clase completa.

Finalmente, al navegador Chrome se le instaló un complemento llamado Screencastify que, en su versión libre, tenía como restricción la duración de 10 minutos por video y un tiempo de generación del archivo respectivo de aproximadamente 1 minuto. La desventaja de este complemento era el tipo de archivo de video generado, en un formato poco común de tipo .webm. Si por alguna causa se deseaba editar alguno de los videos obtenidos, usando MovieMaker que viene incluido en Microsoft Windows, el archivo .webm resultó incompatible con el editor de video lo cual implicaba que primero se debiera convertir a formato .mp4, a través del reproductor multimedia VLC, sin embargo, se decidió asumir este costo y adoptar Screncastify como la herramienta para la producción de video. Todo esto fue depurándose sobre la marcha, a prueba y error.

Existía también el inconveniente de no contar con un lugar ex profeso para la producción de los videos por lo que se trataba de encontrar el sitio donde menos ruido hubiera en el momento de la grabación ya que Screencastify utiliza el micrófono de la computadora, mismo que podía captar el sonido del ambiente, afectando la calidad del audio. 
Una vez obtenido el video, se publicaba en un grupo secreto (denominado así el tipo de grupo con mayor grado de privacidad, al que solo tienen acceso los miembros explícitamente invitados a formar parte de él y no es visible para el público en general) previamente creado en Facebook, integrado por el profesor y los alumnos de la clase. En este grupo, los estudiantes podían visualizar los videos correspondientes a la clase de cada día y hacer preguntas, mismas que eran respondidas por el profesor, quien se conectaba a la hora de clase para tratar de que los alumnos aprovecharan ese tiempo desde la escuela, pero si surgían preguntas en otra hora, igualmente eran válidas y respondidas, sobrepasando las limitaciones de una clase presencial en cuanto a horario, como puede observarse en la Fig. 1.

\section{Q}

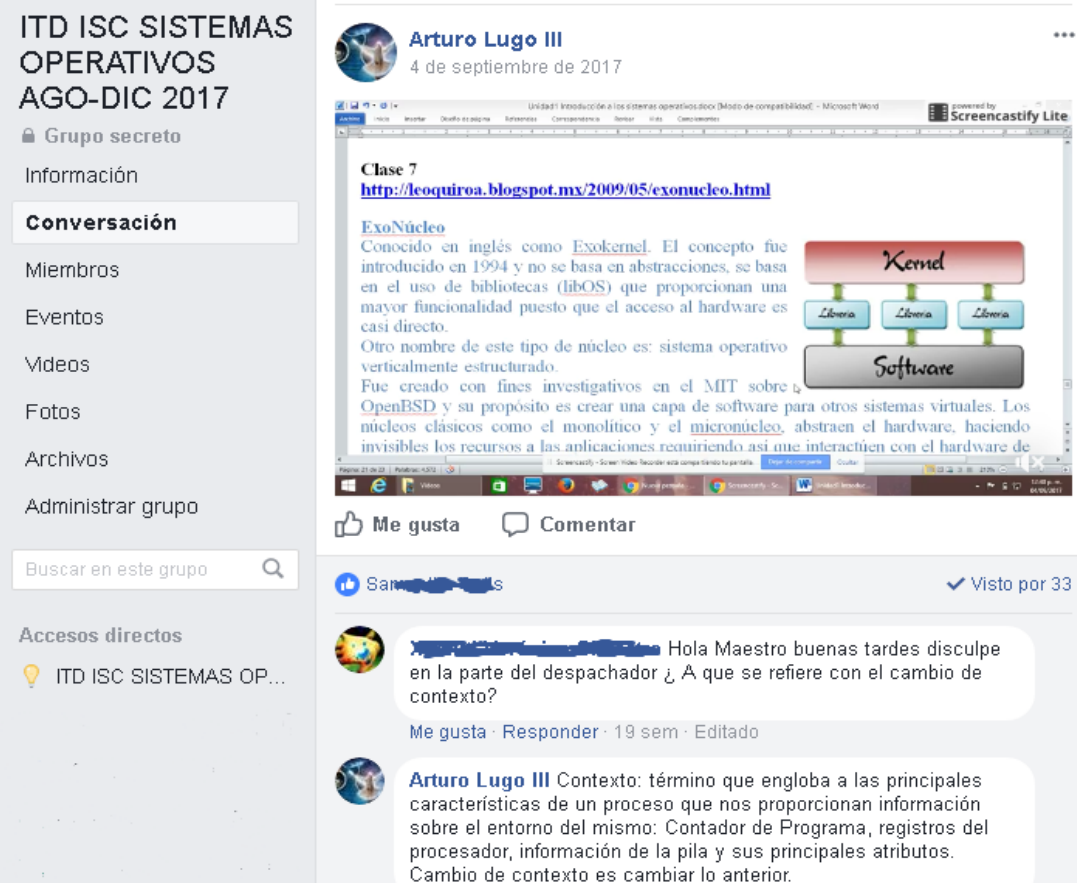

Fig. 1 Ejemplo de publicación de video y resolución de dudas

Fuente: (Lugo Morales, Facebook, 2017) 


\section{Resultados}

La materia de Sistemas Operativos se ha impartido consecutivamente en modalidad presencial desde el periodo agosto-diciembre 2013, es decir, en 9 semestres ininterrumpidos y en ocasiones anteriores no consecutivas, por lo cual hay un registro de calificaciones previas que se pueden contrastar con las obtenidas durante este ejercicio, sin embargo, se hará un comparativo entre las calificaciones obtenidas por el grupo del primer semestre del 2017 y el del segundo, ya que son los que presentaron las circunstancias ya descritas y con la distinción de que el primer periodo fue en su mayoría presencial y el segundo en su totalidad semipresencial.

Se puede observar en la Fig. 2, valores en general favorables en el semestre AgostoDiciembre 2017, con excepción de la unidad 1 que tiene un índice de reprobación muy alto comparado con la misma unidad del semestre Enero-Junio 2017 (11\% vs 42\%). Esto puede atribuirse al desconocimiento del modelo semipresencial en el caso del grupo del segundo semestre de 2017 pero a partir de familiarizarse con este esquema se aprecia una baja en los porcentajes de reprobación.

Habitualmente la unidad 2 presenta un índice de reprobación alto, en muchas ocasiones de más del $50 \%$ ya que incluye, además del examen teórico, el desarrollo de un programa simulador del administrador de procesos de un sistema operativo, lo cual representa una dificultad para la mayoría de los alumnos. En enero-junio 2017 esta unidad fue impartida en modalidad presencial, en agosto-diciembre, en modalidad semipresencial, y los índices de reprobación variaron drásticamente: $82 \%$ vs $44 \%$, y la variación más extrema se presentó en la unidad 3, 96\% vs 3\%.

Para el caso de la unidad 4, se puede ver un comportamiento inverso de $29 \%$ vs $42 \%$, lo cual puede atribuirse a que el modelo no es perfecto y los alumnos pueden encontrar cierta comodidad que los hace confiarse.

A continuación se muestra un ejemplo de esto. Desde que se advirtió por parte del médico la restricción para evitar el contacto con personas que presentaran síntomas de infecciones, se dio la facilidad a los alumnos de presentar proyectos y evaluaciones en equipo, previendo que si algún integrante de algún equipo presentara cierta afectación en su salud que fuera riesgosa para el docente, pudiera ausentarse y el resto del equipo hiciera el trabajo, sin que eso significara que el alumno que no asistía se desentendiera de la materia, es decir, debería seguir al pendiente de lo que sucedía en el desarrollo de las clases.

La anterior facilidad de presentar los exámenes en equipo provocó que, en el caso del segundo semestre de 2017, algunos alumnos se relajaran y se confiaran, porque solo estudiaban "la parte que les correspondía". Al hacerse evidente esta situación, se implementaron algunos cambios, por ejemplo, la aplicación de exámenes individuales y a 
pesar de que se avisó de la posibilidad de que el examen fuera así, aquellos que se confiaron vieron con sorpresa, como resultado de esa comodidad mal entendida, que su preparación para el examen no fue suficiente y su calificación bajó, pero sin llegar a casos exagerados que superaran el $50 \%$ de reprobación.

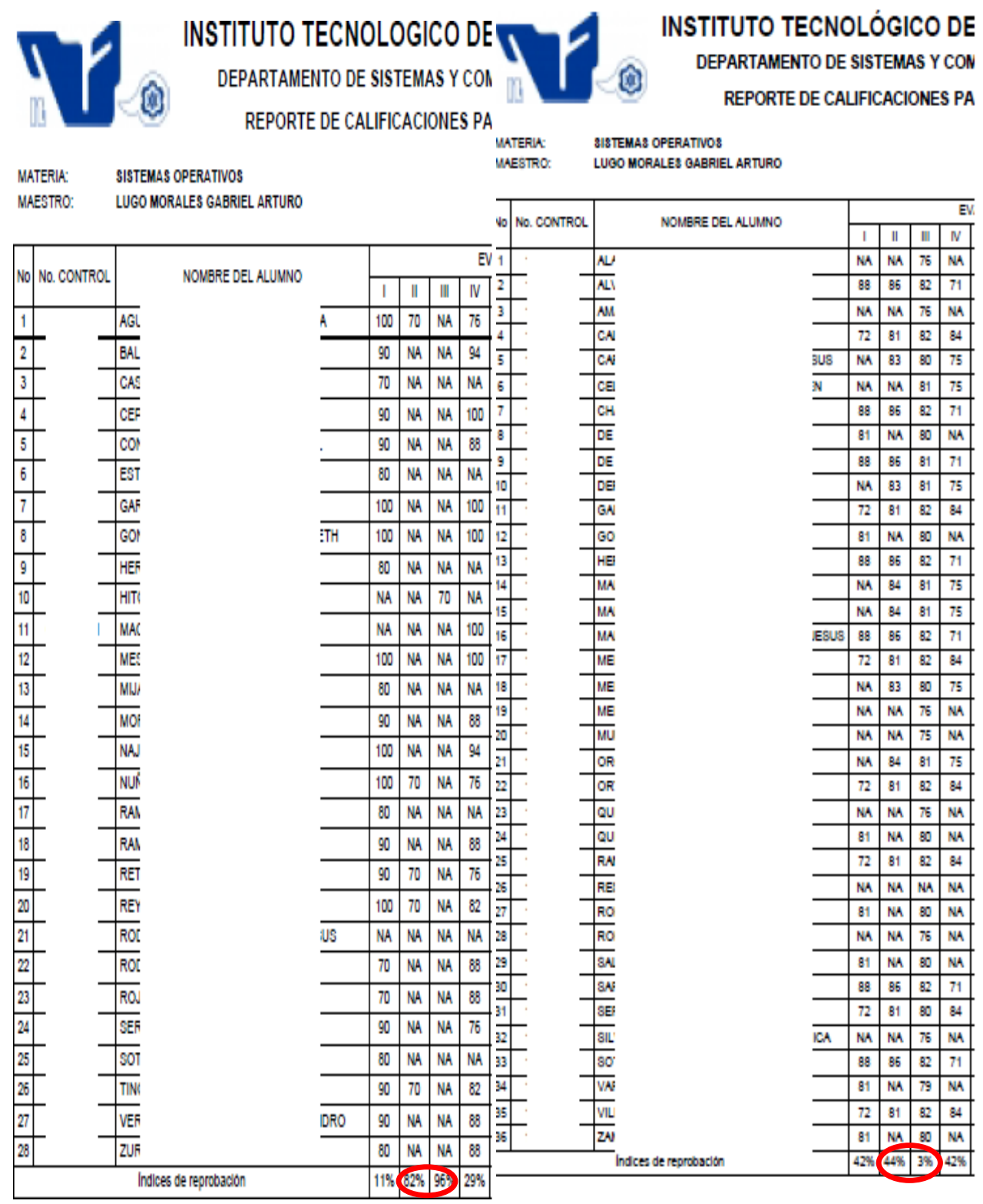

Fig 2. Comparativo de índices de reprobación en los semestres Enero-Junio (Izq.) y Agosto Diciembre (Der.) Fuente siit.itdurango.edu.mx (Instituto Tecnológico de Durango, 2017) 


\section{Conclusiones}

Esta experiencia ha sido útil tanto para el docente como para sus estudiantes pues permitió que se impartieran todos los temas y en la mayoría de los casos, se aprobara la materia, aunque tuvo mucho mejor resultado en la segunda ocasión que se implementó desde el inicio pues se explicaron las condiciones en que la clase se impartiría mientras que en la primera fue una situación emergente y casi finalizando el semestre. Los alumnos requieren un proceso de concientización y adaptación al modelo.

Los resultados obtenidos muestran como ventajas que:

- Un profesor, incapacitado para asistir al aula por cuestiones de enfermedad, puede impartir su clase obteniendo buenos resultados y llevar a la par su tratamiento, lo cual felizmente, le permitió recuperar su salud.

- Se supera la limitación de las clases presenciales en cuanto a tiempo y espacio pues al estar el material en una plataforma que permite que se visualice en cualquier momento y en cualquier lugar (siempre y cuando haya conexión a Internet y el equipo necesario), ya no se restringe al estudiante a tomar la clase en un lugar específico ni en un momento específico, ni al docente a impartir la cátedra y resolver las dudas en esos mismos términos.

- Al dejar los materiales en un repositorio que puede ser consultado en todo momento, el alumno puede repasar la clase las veces que requiera, lo cual no se logra en la modalidad presencial donde el profesor imparte cada tema solamente en una ocasión.

Los resultados obtenidos sugieren que uno de los principales cuidados que se deben tener es que, por más innovador que sea un método de enseñanza, por más tecnología que se disponga y por más facilidades que se ofrezcan, si los participantes no realizan la parte que les corresponde de manera responsable, los resultados no serán los deseados.

Un valor agregado de la estrategia diseñada, que no se tenía contemplado al inicio es que, al tener un grupo en Facebook y dado que la mayoría de los estudiantes poseen actualmente un Smartphone, se puede mantener comunicación casi al instante entre todos los integrantes del grupo, lo cual es de gran utilidad para notificar avisos, como sucedió en alguna ocasión que se tenía programado un examen y por situaciones no previstas, el edificio donde se ubica el salón de clases estaría cerrado. Para evitar que los alumnos se trasladaran desde su casa hasta el Instituto, se les avisó la reprogramación del examen; esa comunicación también sirvió para generar un buen ambiente, por ejemplo, al felicitar por su cumpleaños a algún miembro. 
Como comentario final, se muestra que, a pesar de las adversidades, se pueden aprovechar los recursos tecnológicos para solventar esas dificultades y sacar adelante los proyectos, esto sin que se pretenda exaltar al docente en cuestión, por el contrario, la mayor enseñanza que esta experiencia le ha dejado es que, siendo una situación difícil donde incluso hubo un desahucio por parte de un oncólogo antes de iniciar el tratamiento, el profesor ante su imposibilidad de resolución de una situación tan grave y como creyente de la fe cristiana se permite citar como parte final de este trabajo de la carta de Pablo a los Filipenses, en el capítulo 4, el verso 13, de la Biblia en su versión Dios Habla Hoy: "A todo puedo hacerle frente, gracias a Cristo que me fortalece" (Meyers, 2017)."

\section{Referencias}

Concha Vergara, M. H. (mayo-agosto de 2014). E-learning. La revolución educativa. Enl@ce Revista Venezolana de Información, Tecnología y Conocimiento [en línea], 11(2), 116.

Durán, E., Costaguta, R., \& Gola, M. (diciembre de 2011). El modelo B-learning implementado en la asignatura simulación. RIED. Revista Iberoamericana de Educación a Distancia, 14(2), 149-166.

Instituto Tecnológico de Durango. (2017). Sistema Integral de Información Tecnológica. Obtenido de siit.itdurango.edu.mx

Lugo Morales, G. A. (4 de septiembre de 2017). Facebook. Obtenido de https://www.facebook.com/groups/1946892902251867

Lugo Morales, G. A., \& González Bañales, D. L. (2016). Modelo de evaluación basado en la simulación de sistemas de pago de salarios: "Día estudiado, día pagado". 3er. Congreso Internacional de Innovación Educativa Memorias CIIE 2016, 619.

Maldonado, E., \& Juárez, J. I. (9 de agosto de 2006). De profesor a asesor. Obtenido de Coordinacion de Educación a distancia UNAM: http://cad.cele.unam.mx:8080/RD3/prueba/pdf/maldonado.pdf

Meyers, R. (2017). E-Sword. Franklin, Tennessee, Estados Unidos de América.

Mirete, A., Maquilón, J., \& Sánchez, M. (2014). Uso de Screencast como Herramientas para la Introducción de Asignaturas Universitarias. En J. Maquilón, \& O. Noelia, Investigación e innovación en formación del profesorado (págs. 624-625). Murcia, España: Ediciones de la Universidad de Murcia.

Mogollón, L. (2016). Metodología de Sistematización de Experiencias Educativas Innovadoras. Lima, Perú: Cartolan E.I.R.L. 